\title{
Wearable recording video technology for surgical training in living donor liver transplantation
}

\author{
Shin Ae Lee, Jeong-Moo Lee, Kyung-Suk Suh, Suk-Kyun Hong, \\ Jae-Hyung Cho, Nam-Joon Yi, and Kwang-Woong Lee \\ Department of Surgery, Seoul National University College of Medicine, Seoul, Korea
}

\begin{abstract}
Backgrounds/Aims: As the development of surgical video recording technologies, educational videos have become widely utilized for trainee education. However, the current forms of surgical video are limited because they do not show all the roles of the participants. Aim of this study is to make optimal training material about living donor liver transplantation for residents and fellows using wearable recording system. Methods: Three video clips about procedure of liver transplantation were made. A head mount was used to fix the camera on the surgeon or assistant's head. Anastomosis of vessels, bench operation and trocar insertion for laparoscopic donor hepatectomy were recorded. Each video clips were edited including indicators, subtitles, and narration. The edited videos were shown to 20 General Surgery trainees (18 residents, 2 fellows) and we received feedback. The results of the questionnaire were quantitatively analyzed to show how efficient and informative it is compared to existing educational materials. Results: Sixteen of the 20 trainees $(80 \%)$ responded that this video helped them improve their surgical skills. Eighteen trainees $(90 \%)$ responded that they gained new knowledge through this video. Sixteen trainees $(80 \%)$ responded that the action camera image material was more educational than existing text-based and video-based materials, with an average score of 8.5 and 6.5 (action camera materials vs. text-based materials, respectively). Conclusions: A head-mounted action camera video recording system is a good model for making high-quality educational video modules and can be a useful teaching tool for living donor liver transplantation. (Ann Hepatobiliary Pancreat Surg 2020;24:38-43)
\end{abstract}

Key Words: Training; Liver transplantation; Medical education

\section{INTRODUCTION}

Living donor liver transplantation is a complicated and meticulous operation; therefore, repeated participation in and observation of surgery are necessary for trainees to learn the entire process. However, in many countries, liver transplantation is usually performed only at large-scale specialized medical centers. Therefore, in small hospital settings, opportunities are extremely rare for trainees to actively observe liver transplantation. Even at specialized centers, there are limited opportunities for residents to directly participate in liver transplantation, and such opportunities are generally offered to chief residents or subspecialty fellows. In addition, because residents' work hours are restricted to 80 hours/week, and the length of general surgery residency in South Korea has recently been adjusted to 3 years instead of 4 years, residents have only a limited amount of time to gain experience in the surgical field. ${ }^{1}$ Such constraints are true not only for residents but also for fellows, as the majority of their work involves perioperative patient care rather than direct participation in liver transplantation surgery.

Therefore, surgeons in training are in need of materials to help them learn efficiently and effectively within the limited surgical residency period. Such materials need to be developed not only for liver transplantation but also for many other surgical procedures; therefore, this issue should be discussed in the entire general surgery department. $^{2-7}$

The purpose of this study was to evaluate the feasibility and efficacy of action cameras for surgical training for liver transplantation and to suggest future roles of wear-

Received: September 18, 2019; Revised: October 7, 2019; Accepted: October 8, 2019

Corresponding author: Jeong-Moo Lee

Department of Surgery, Seoul National University College of Medicine, 101 Daehak-ro, Jongno-gu, Seoul 03080, Korea Tel: +82-2-2072-2817, Fax: +82-2-766-3975, E-mail: lulu5050@naver.com

Copyright (C) 2020 by The Korean Association of Hepato-Biliary-Pancreatic Surgery

This is an Open Access article distributed under the terms of the Creative Commons Attribution Non-Commercial License (http://creativecommons.org/ licenses/by-nc/4.0) which permits unrestricted non-commercial use, distribution, and reproduction in any medium, provided the original work is properly cited. Annals of Hepato-Biliary-Pancreatic Surgery • pISSN: 2508-5778 - eISSN: 2508-5859 


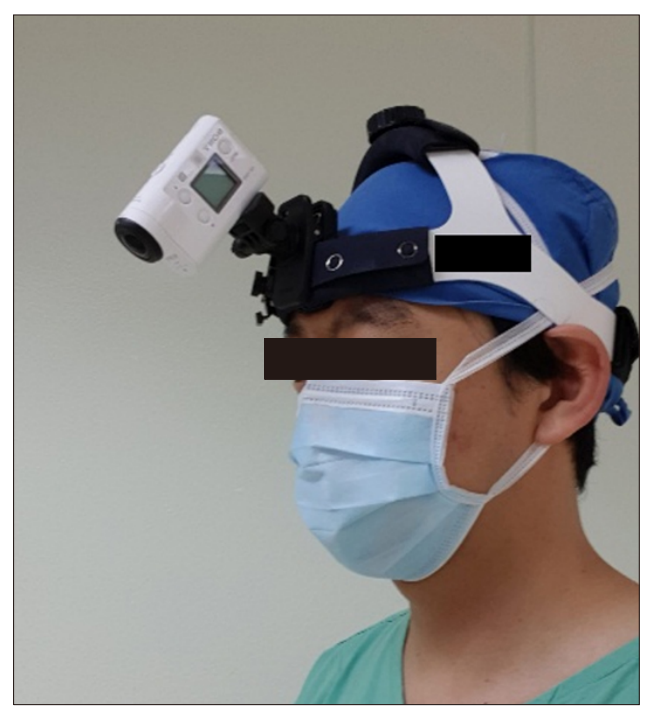

Fig. 1. Head mount to fix the action camera (SONY FDR-X3000R) on the head of a surgeon or assistant.

able devices in surgery.

\section{MATERIALS AND METHODS}

This study was approved by the Institutional Review Board (IRB) of Seoul National University Hospital (IRB no. 1906-079-1040), and the IRB waived the need for informed consent from the anonymously surveyed residents.

\section{Recording device}

From January 2018 to December 2018, 6 videos of routine cases of living donor liver transplantation were recorded by an action camera FDR-X3000R (SONY Corporation, Japan). Although prior studies usually have evaluated the efficacy of popular wearable devices including GoPro (GoPro Inc, San Mateo, CA, USA) or Google Glass (Google Inc, Mountain View, CA, USA), our study was the first to apply the SONY FDR-X3000R wearable action camera. We selected this camera because it can provide more stable video clips with its electrical motion stabilizer. A head mount was used to fix the camera on the head of a surgeon who was experienced in performing the procedure (Fig. 1). Each video clip was recorded in $4 \mathrm{k}$ high definition quality.

\section{Indications for educational clip selection}

We divided each procedure of liver transplantation into segments to create the educational video clips. We finally made 3 educational clips from all recorded video files. The content of the first educational video included hepatic vein anastomosis, portal vein anastomosis, hepatic artery anastomosis, and bile duct anastomosis, which were recorded from the first assistant's viewpoint. Because residents usually join the operation as a first assistant, many residents want to become familiar with the real practices in the operating room. The second clip showed the bench operation for middle hepatic vein reconstruction because it is a stable procedure with little movement. The last educational clip showed the making of appropriate port sites for laparoscopic donor hepatectomy for clinical fellows, as determining the optimal trocar site for donor hepatectomy is a very difficult and stressful job for fellows or junior faculty. Our institution performs the largest number of laparoscopic donor resections in worldwide. Therefore, we can provide a good educational module for optimal port placement for anyone who wants to learn the techniques for laparoscopic donor hepatectomy. ${ }^{8}$ This educational clip might be helpful for those determining the optimal trocar site for laparoscopic donor hepatectomy. We provided all unedited videos to the trainees who wanted the full video and also provided edited clips for trainees who wanted the edited version.

\section{Description of each educational clip}

First assistant's view of the anastomosis

Liver implantation procedure (hepatic vein anastomosis, portal vein anastomosis, reperfusion, hepatic artery anastomosis, and bile duct anastomosis) clips were prepared. This shot focused on the movements of both hands of the first assistant, and descriptions of important anatomical structures and the assistant's movements were added using still images (Fig. 2A). It is important to retract the opposite side of the leaflet during hepatic vein and portal vein anastomosis. The counter traction points were well described in these video clips.

\section{Bench surgery}

After explantation, hepatic artery perfusion was done immediately through a $10 \mathrm{cc}$ syringe with a $24 \mathrm{G}$ IV catheter. Then, graft perfusion was done via portal vein catheterization. After bile duct irrigation and trimming of the right hepatic vein and portal vein, a reconstruction procedure of the middle hepatic vein was performed by attaching V5, V8 to the right hepatic vein with 6-0 Prolene 

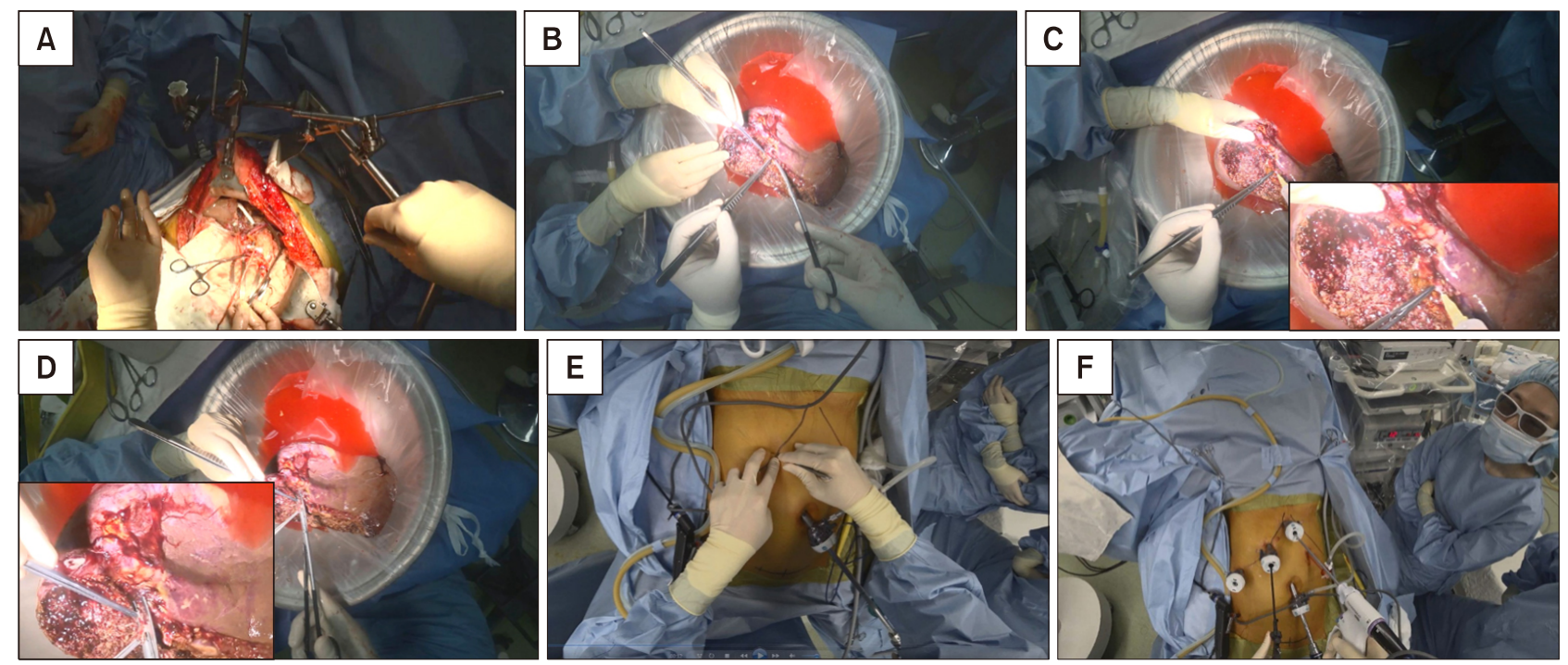

Fig. 2. (A) 1st assistant view for hepatic vein anastomosis. (B) Bench operation (surgeon view). (C) Bench operation (magnified view for artery perfusion). (D) Bench operation (magnified view for portal vein stump trimming). (E) Making trocars for pure laparoscopic donor hepatectomy (surgeon view). (F) Pure laparoscopic donor hepatectomy (surgeon's view).

(Ethicon, USA) and artificial expanded polytetrafluorethylene using an all-in-one technique. However, all these processes are clearly observed only from the operator's view of the bench work, so we showed magnified scenes of catheterization for perfusion using a video editing program (Fig. 2B-D).

Making trocars for laparoscopic donor hepatectomy

The trocar port system for laparoscopic living-donor hepatectomy was made by the liver transplantation fellows and junior faculty staff, and they usually received feedback from senior experts during the operation. We recorded the surgical field for port placement using the head-mounted camera. These recordings were reviewed after the operation and correlated to the senior experts' comments (Fig. 2E, F). After liver mobilization by division of the coronary and triangular ligaments, the liver moves downwards under the costal margin. The change of the liver position made it difficult to use the early inserted trocars.

We made it our practice to place the ports one by one and not all at once. Sequential port placement allowed accurate identification of the liver borders and location after gradated liver mobilization. We included some additional pictures to guide optimal port placement in this video.

Editing

Sony Vegas Pro ver. 13.0 (Sony Corporation, Japan) was used for editing tools, and all video clips were edited for trainee education including indicators, subtitles, and narration. The recorded video clips were high quality, which allowed for zooming and clear visualization of the surgical anatomy. Indicators and subtitles were added by an experienced operator and assistant. The video clips were revised after receiving initial feedback from the trainees.

Survey

It is not easy to measure the educational effects of educational materials. To determine the educational effect for an operator, we usually use learning curve analysis via operative time and complication rate. However, it is not easy to objectify improvements in the proficiency of resident assistants. Therefore, we compared the usefulness of the material through an educational satisfaction survey administered to the trainees, and we investigated the advantages and weaknesses of each of the materials through their feedback. The survey comprised 7 questions that evaluated the quality of the materials and the satisfaction of the trainees. The items on the satisfaction survey of the surgical residents are shown in Table 1.

\section{RESULTS}

During the period between August 2018 and October 2018, 20 trainees were enrolled this educational program. They watched each video clip and responded to the sat- 
Table 1. Questionnaire survey form evaluating educational materials

\begin{tabular}{ll}
\hline \multicolumn{1}{c}{ Questionnaire } & Yes \\
\hline 1 & Did this material help you improve your surgical skills? \\
2 & Based on this video material, have you gained new knowledge \\
(compared to other educational materials)? \\
3 & After this training, I could perform these skills independently as a first assistant. \\
4 & How well prepared was this video supplement - were the time, indicators, \\
& and figures appropriate? \\
& Reason (open question) \\
6 & Is it correlated with a real operation? \\
Is it better than previous text-based or other video materials? \\
$\quad$ (Score all materials on a scale of 1-10.) \\
If yes: reasons \\
If no: reasons \\
Other feedback (open question)
\end{tabular}

Table 2. Results of learning efficiency by questionnaire survey

\begin{tabular}{lrr}
\hline Learning efficiency & Yes & No \\
\hline Did this material help you improve your surgical skills? & $16(80 \%)$ & $4(20 \%)$ \\
Based on this video material, have you gained new knowledge? & $18(90 \%)$ & $2(10 \%)$ \\
After this training, I could perform these skills independently as a first assistant. & $10(50 \%)$ & $10(50 \%)$ \\
\hline
\end{tabular}

Table 3. Evaluation of the education video quality by questionnaire survey

\begin{tabular}{lcc}
\hline \multicolumn{1}{c}{ Video quality } & Yes & No \\
\hline Appropriation of contents & $12(60 \%)$ & $8(40 \%)$ \\
Appropriation of indicators and explanations & $12(60 \%)$ & $8(40 \%)$ \\
Correlation of actual operations and videos & $18(90 \%)$ & $2(10 \%)$ \\
More educational than existing materials? & $16(80 \%)$ & $4(20 \%)$ \\
\hline
\end{tabular}

isfaction survey. Twelve trainees were vice chief residents, 6 trainees were chief residents, and 2 trainees were fellows.

\section{Questionnaire for evaluating learning efficiency}

Sixteen of the 20 trainees $(80 \%)$ responded that this video helped them improve their surgical skills. Eighteen trainees $(90 \%)$ responded that they gained new knowledge through this video. Ten trainees (50\%) responded that they would be able to perform the surgery independently after viewing the video (Table 2). Twelve of 20 trainees $(60 \%)$ stated that the contents were appropriate, and 6 trainees $(30 \%)$ answered that they wanted somewhat more content. Eight of 20 trainees (40\%) preferred the full-length video, and 12 trainees $(60 \%)$ stated that the edited video was more educational. Most of the trainees preferred the edited one because of the long running time.
However, in the case of the bench operation, most agreed that it would be better to watch the whole procedure and that the full video would be better.

Twelve trainees $(60 \%)$ reported that the indicators and explanations were appropriate, and 18 trainees (90\%) stated that the actual operation and video were well correlated. Sixteen trainees $(80 \%)$ responded that the action camera image material was more educational than existing text-based and video-based materials, with an average score of 6.5 and 8.5 (text-based materials vs action camera materials respectively) (Table 3 ). Ten percent of trainees ( 2 of 20$)$ responded that the action camera materials were similar to existing video material.The respondents who gave positive feedback reported that the videos were helpful because they had the impression that they were participating in the surgery directly. They also stated that the movements of the two hands were clearly 
visible, and this was more helpful. On the other hand, some expressed the opinion that the video was difficult to watch because it moved when the operator's head moved.

\section{DISCUSSION}

Most educational materials for surgical residents depend on textbooks and descriptions by the senior trainees. However, text-based educational materials have inherent limitations because narrative methods is different depending on the narrator, and it is difficult to fully understand the actual three-dimensional surgical field. As a result, demand for video instructional materials that have been recorded during actual surgeries in increasing recently. With the development of surgical cranes and laparoscopic surgery, more information can be transmitted through video than text-based materials. ${ }^{9}$ However, most videos are confined to the operator's main field, so it is difficult to observe the hand movements of the first assistant. Moreover, owing to discrepancies between the viewpoints of the operator, assistant, and surgical crane, it is occasionally difficult to accurately capture the surgical field because the head of the surgeon or the assistant blocks the view. ${ }^{10}$ Another problem with existing surgical video is that differences in resolution may occur depending on each hospital's equipment.

In order for the first assistant to fully understand his or her role before surgery and to undergo video training, operative video taken from the first assistant's viewpoint as well as that from the operator's viewpoint is needed. ${ }^{11}$ As mentioned above, since the surgical crane or the laparoscopic camera record the surgical field from a third position, an action camera is necessary to produce educational videos from the first assistant's point of view.

The action camera can solve the problems of existing educational materials mentioned above. The video can be taken from the observer's point of view, and this technology is highly portable and can produce high-quality images. Moreover, by sharing experts' experiences, trainees can increase their indirect experience and understand more about the surgical procedure.

The results of the questionnaire showed that the trainees' satisfaction was high, and indirect learning was possible through indirect experience. The action camera video seemed to have provided satisfactory training for the actual operation. To know what trainees have to do in surgery played a major role because they could see exactly what to do with their hands. Therefore, we believe that this device-based technique would be useful for all operations, not just liver surgery. It can be used not only for its educational role but also as an in-training test tool and for open feedback.

But this study has limitations, when the head is shaken heavily, low-quality images can be recorded. And even we chose Sony over the Go-Pro to reduce dizziness, because the model of SONY has motion stabilization itself, however, someone still felt nausea and dizziness during watching the training video. However, in most cases, trainees watched the recorded video without much difficulty, and only a few viewers complained of dizziness. Additionally, bench surgery has little motion and has rarely been associated with dizziness. Trainee dizziness while viewing the video is a minor limitation because only high-quality video is retained after editing. Another limitation is that it is somewhat difficult to record for a long time owing to battery problems. However, we made an effort to solve this problem by choosing a battery exchange type of camera that was different from other products. Thus, the surgical field in the video was somewhat wide, but we as we used $4 \mathrm{k}$ picture quality, we were able to enlarge the necessary parts as described above without deterioration of the image quality.

Until now, there has been no standard survey tool to quantify residents' satisfaction. ${ }^{1}$ Therefore, our institution has tried to evaluate the satisfaction of its residents by creating its own survey tool, but there is no standard comparison tool. However, we showed that the educational materials created with the action camera were better than the existing materials in terms of information provision and image quality. If a high-quality survey tool will be developed and the use of educational videos recorded by action camera will be expanded in near future, we can evaluate more trainee and show well the better effect of this tool.

This reports of our initial experience with implementing an action camera for training, and there is currently no such report regarding surgical trainee education. We are responsible for training surgeons within 3 years in the upcoming 3-year surgery resident training system with only 
80 hours of training time per week. Because it is not easy to learn all the surgical techniques in such a limited training time, resident education using high-quality surgical videos will become more important in the future.

In conclusion, the development of educational materials using an action camera has the potential to be used not only in liver transplantation but also in the entire surgical field. If this indirect experience enhances the quality of education, it will be a good way to solve the problems caused by little opportunity to join the real surgical filed and limited time of residency training.

\section{ORCID}

Shin Ae Lee: https://orcid.org/0000-0002-1157-0442

Jeong-Moo Lee: https://orcid.org/0000-0001-7806-8759

Kyung-Suk Suh: https://orcid.org/0000-0002-9535-7349

Suk-Kyun Hong: https://orcid.org/0000-0002-0020-6215

Jae-Hyung Cho: https://orcid.org/0000-0003-0188-8278

Nam-Joon Yi: https://orcid.org/0000-0002-5467-425X

Kwang-Woong Lee: https://orcid.org/0000-0001-6412-1926

\section{AUTHOR CONTRIBUTIONS}

Conceptualization: JML, KSS. Data curation: SAL, SKH, JHC. Formal analysis: SAL, JML.

Methodology: JML, NJY. Project administration: JML. Visualization: SAL, JML, SKH, JHC.

Writing - original draft: SAL, JML. Writing - review \& amp; editing: JML, KSS, NJY, KWL

\section{REFERENCES}

1. Moore MD, Abelson JS, O'Mahoney P, Bagautdinov I, Yeo H, Watkins AC. Using GoPro to give video-assisted operative feedback for surgery residents: a feasibility and utility assessment. J Surg Educ 2018;75:497-502.

2. Ganry L, Sigaux N, Ettinger KS, Salman SO, Fernandes RP. Modified GoPro Hero 6 and 7 for intraoperative surgical recording-transformation into a surgeon-perspective professional quality recording system. J Oral Maxillofac Surg 2019;77:1703. e1-1703.e6.

3. Ortensi A, Panunzi A, Trombetta S, Cattaneo A, Sorrenti S, D'Orazi V. Advancement of thyroid surgery video recording: a comparison between two full HD head mounted video cameras. Int J Surg 2017;41 Suppl 1:S65-S69.

4. Singh P, Aggarwal R, Tahir M, Pucher PH, Darzi A. A randomized controlled study to evaluate the role of video-based coaching in training laparoscopic skills. Ann Surg 2015;261:862-869.

5. Jensen AR, Wright $A S$, Kim $S$, Horvath KD, Calhoun KE. Educational feedback in the operating room: a gap between resident and faculty perceptions. Am J Surg 2012;204:248-255.

6. Nair AG, Kamal S, Dave TV, Mishra K, Reddy HS, Della Rocca $\mathrm{D}$, et al. Surgeon point-of-view recording: Using a high-definition head-mounted video camera in the operating room. Indian J Ophthalmol 2015;63:771-774.

7. Bonrath EM, Dedy NJ, Gordon LE, Grantcharov TP. Compr ehensive surgical coaching enhances surgical skill in the operating room: a randomized controlled trial. Ann Surg 2015;262: 205-212.

8. Lee JM, Shehta A, Suh KS, Hong SK, Yoon KC, Cho JH, et al. Guidance for optimal port placement in pure 3-Dimensional laparoscopic donor right hepatectomy. Liver Transpl 2019;25: 1714-1722.

9. Ahmet A, Gamze K, Rustem M, Sezen KA. Is video-based education an effective method in surgical education? A systematic review. J Surg Educ 2018;75:1150-1158.

10. Lee CK, Kim Y, Lee N, Kim B, Kim D, Yi S. Feasibility study of utilization of action camera, GoPro Hero 4, Google Glass, and Panasonic HX-A100 in spine surgery. Spine (Phila Pa 1976) 2017;42:275-280.

11. Rose JS, Waibel BH, Schenarts PJ. Disparity between resident and faculty surgeons' perceptions of preoperative preparation, intraoperative teaching, and postoperative feedback. J Surg Educ 2011;68:459-464. 